This item was submitted to Loughborough's Research Repository by the author.

Items in Figshare are protected by copyright, with all rights reserved, unless otherwise indicated.

\title{
Techniques for modelling the frequency of explosions on offshore platforms
}

PLEASE CITE THE PUBLISHED VERSION

PUBLISHER

(C) IMechE / Professional Engineering Publishing

LICENCE

CC BY-NC-ND 4.0

REPOSITORY RECORD

Foster, K.J., and J.D. Andrews. 2008. "Techniques for Modelling the Frequency of Explosions on Offshore Platforms". figshare. https://hdl.handle.net/2134/3819. 
This item was submitted to Loughborough's Institutional Repository (https://dspace.lboro.ac.uk/) by the author and is made available under the following Creative Commons Licence conditions.

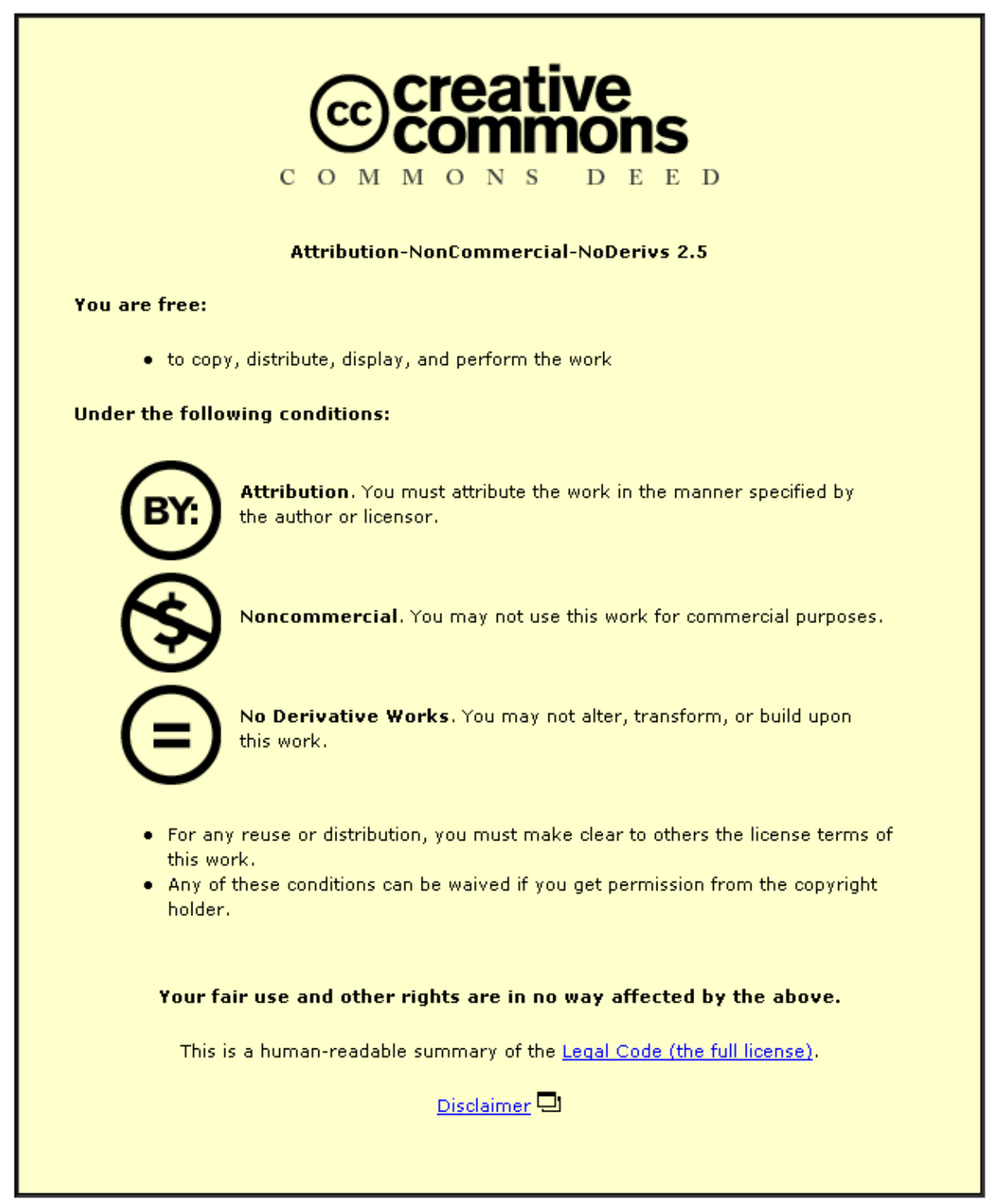

For the full text of this licence, please go to: http://creativecommons.org/licenses/by-nc-nd/2.5/ 


\title{
Techniques for modelling the frequency of explosions on offshore platforms
}

\author{
K J Foster and J D Andrews* \\ Department of Mathematical Sciences, Loughborough University, Loughborough, UK
}

\begin{abstract}
This paper describes a scheme by which the explosion frequencies and resulting overpressures can be predicted for modules on an offshore platform. Given a leak of gas, the success and failure of the isolation, blow-down and deluge systems, which are designed to activate following a leak to mitigate the consequences, are considered. The frequency of each possible response to the leak is predicted using a conventional event tree and fault tree approach. Each scenario has a potential escape inventory, which together with the hole size distribution provides the escaping mass flowrate. Once the flowrate into the module is established, the wind conditions are used to determine the gas in air concentration build-up. The concentration profile with respect to time is linked to the ignition source probabilities, from which calculations can be made to assess the likelihood that an explosion will occur within a specific concentration range.

The initial models assume that the gas concentration at any specific time is uniform within the locality of the leak. The work presented in this paper proposes an approach that determines the explosion probability dependent on the volume fraction of gas within the module and the likelihood of encountering an ignition source.
\end{abstract}

Keywords: explosion modelling, risk assessment, offshore

\section{INTRODUCTION}

The Piper Alpha disaster in 1988 led to reforms in offshore safety regulations. As a result of the public enquiry led by Lord Cullen [1], offshore operators are required to demonstrate that the potential major hazards of the installation and risks to personnel have been identified and appropriate controls provided. On an offshore platform, two of the major hazards are fires and explosions resulting from an accidental hydrocarbon release. The overpressure generated during an explosion can threaten the integrity of the platform structure. It is therefore important to be able to estimate the overpressures generated, should an explosion occur, and to predict the frequency of such an event.

A methodology has been developed to predict the frequency of explosions of different magnitudes occurring in a module on an offshore platform [2]. This methodology combines established risk assessment techniques, such as event tree analysis [3] and fault tree analysis [4], with fluid flow modelling $[\mathbf{5}, \mathbf{6}]$. Fault tree

The MS was received on 10 June 1998 and was accepted after revision for publication on 23 March 1999.

*Corresponding author: Department of Mathematical Sciences, Loughborough University, Loughborough, Leicestershire LE11 3TU, UK. and event tree methods are used to determine the frequencies of occurrence of all possible scenarios resulting from a leak of gas. Each scenario evolves from the initial gas leak from a process section that can be isolated. The event tree branch points determine whether the gas detection system functions, the relevant isolation and blow-down valves function and the deluge system activates. A distribution of leak hole sizes is considered. Using fluid flow modelling, the gas release rate is computed, allowing the variation in the module gas concentration with time to be calculated. The frequency of an explosion is calculated by combining the time that the concentration is between the flammable limits with the predicted ignition rate. The magnitude of the overpressures produced from an ignition are determined from an empirical relationship produced by British Gas described by Catlin et al. [7] and Gregory and Johnson [8].

This paper presents two methods for calculating the frequency of an explosion. The first method assumes that the gas in air concentration within the module is uniform; therefore an ignition source occurring at any position will, given a flammable concentration, cause an explosion. The ignition rate in this case is taken to be constant. The second method requires the use of 


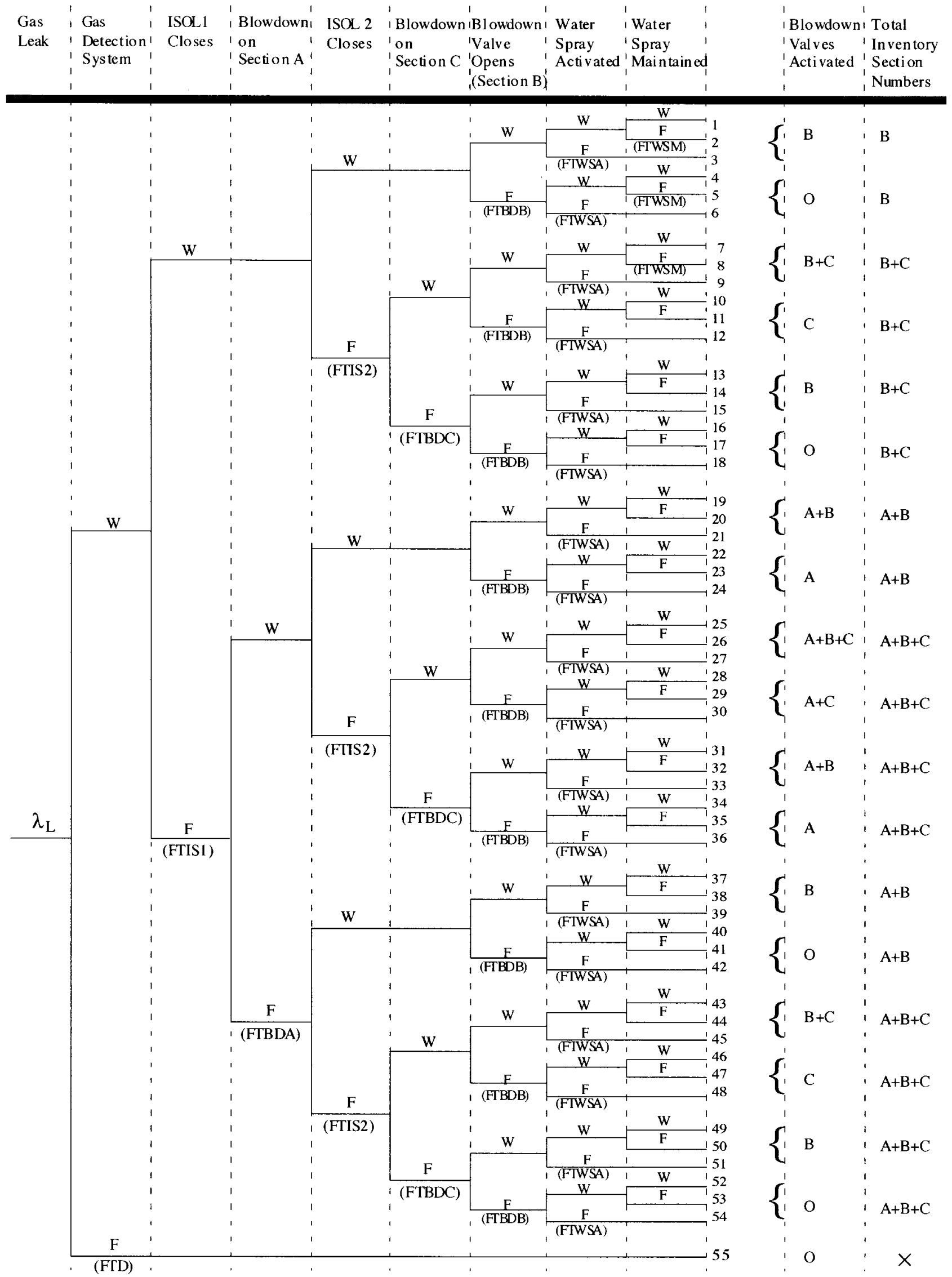

Fig. 1 Event tree identifying all possible outcomes following a gas leak on a specified section 
computational fluid dynamics (CFD) to determine the concentration distribution throughout the module; such correlations mean that it is possible to produce a relationship between the volume of the gas cloud within set concentration limits and time. This method determines the explosion frequency dependent on the volume fraction of gas within the module and the likelihood of encountering an ignition source.

\section{FREQUENCIES OF EXPLOSIONS}

The frequency of an explosion depends on:

(a) the frequency of leakage,

(b) the frequency of ignition,

(c) the time of detection of the gas concentration,

(d) the availability of explosion-mitigating systems,

(e) the reaction time of explosion-mitigating systems,

(f) the availability of a blow-down system,

(g) the availability of a gas detection system,

(h) the availability of an isolation system,

(i) the source strength of leakage,

(j) the pressure-time history of the section,

(k) the ventilation rate and

(1) the concentration-time history of the gas-air mixture.

Event trees are used to determine all possible scenarios. The event tree diagram represents the response to the initiating event (gas leak) of the gas detection, isolation, blow-down and water spray systems. For example, see the event tree shown in Fig. 1 for a simple situation where the leak has occurred on a section bounded by two isolation valves and contains a single blow-down valve. Following a significant leak, the detection system should respond by activating the protection systems. Isolation valves will limit the leak inventory of gas, if operating correctly. Blow-down valves allow the gas to be burnt at the flare, reducing the mass of gas and pressure in the leaking section. Water spray systems activate to limit the consequences of an explosion by reducing the overpressures produced. Fault trees are constructed to represent the causes of the failure of each isolation valve, blow-down valve, detection system and water spray. Hence the frequency of each scenario occurring is obtained. The event tree constructed in Fig. 1 has binary branching, indicating that the detection, isolation, blow-down and deluge are considered to be either totally successful or a total failure. The method is flexible and can allow multiple branching to incorporate partial failures. However, from a practical viewpoint, accurate probabilities of such events are rarely available and it is felt that considering such eventualities as total failures, i.e. pessimistically, is the most sensible approach.

The state of the isolation and blow-down valves determines the amount of gas available to leak and the pressure within the section. The behaviour of the fluid is modelled to obtain the mass flowrate of gas into the module. The ventilation system on the platform is assumed to be natural, dominated by the wind speed and direction; therefore a distribution of ventilation rates is considered. Utilizing the appropriate ventilation rate, a concentration-time history of gas in air is obtained for the module.

Ignition may only occur if the concentration of gas is between the flammable limits. For natural gas the lower flammable limit (LFL) is approximately 5 per cent and the upper flammable limit (UFL) is 15 per cent. If perfect mixing occurs and the gas concentration within a fixed volume is assumed constant, then the concentration time history may be one of three forms. The situation shown in Fig. 2a could not result in an explosion, as the concentration of gas does not build up to the flammable region. In Fig. 2b, ignition may occur between $T_{1}$ and $T_{4}$. However, in Fig. 2c, ignition may occur in two time periods, between $T_{1}$ and $T_{2}$, while the concentration is rising and again between $T_{3}$ and $T_{4}$ as it falls back between the flammable limits.

There are two categories of explosions dependent on the availability of the water spray system: mitigated and unmitigated. Mitigated explosions result in lower overpressures; see Fig. 3 taken from Catlin et al. [7]. Mitigated explosions occur when the water spray is operational, for this we must consider the following cases:
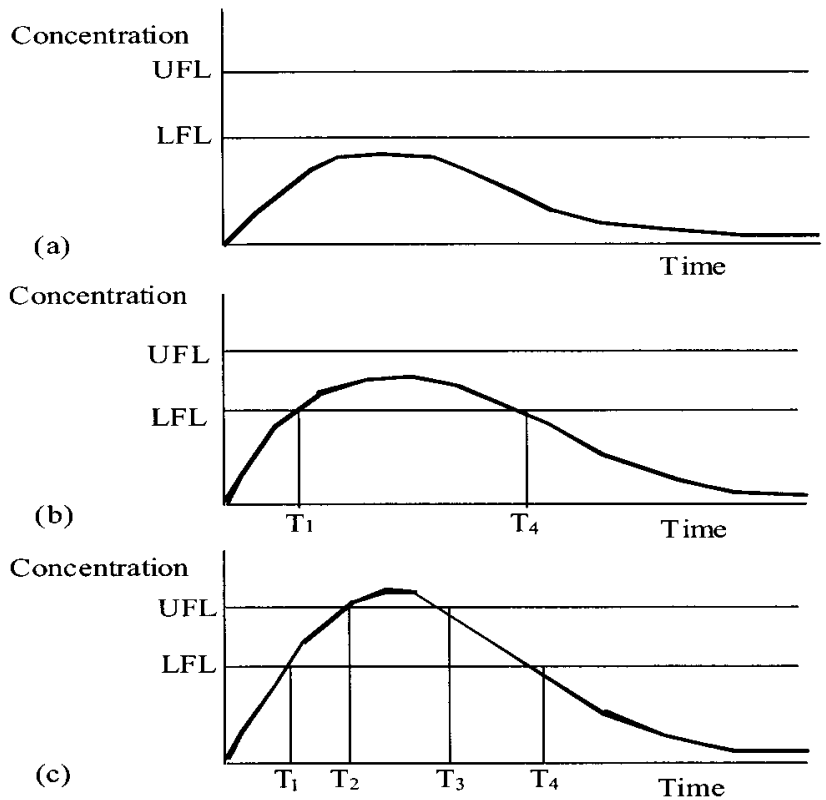

Fig. 2 Typical gas concentration-time histories: (a) the gas concentration is always below the LFL; (b) the gas concentration exceeds the LFL but not the UFL; (c) the gas concentration exceeds the UFL 


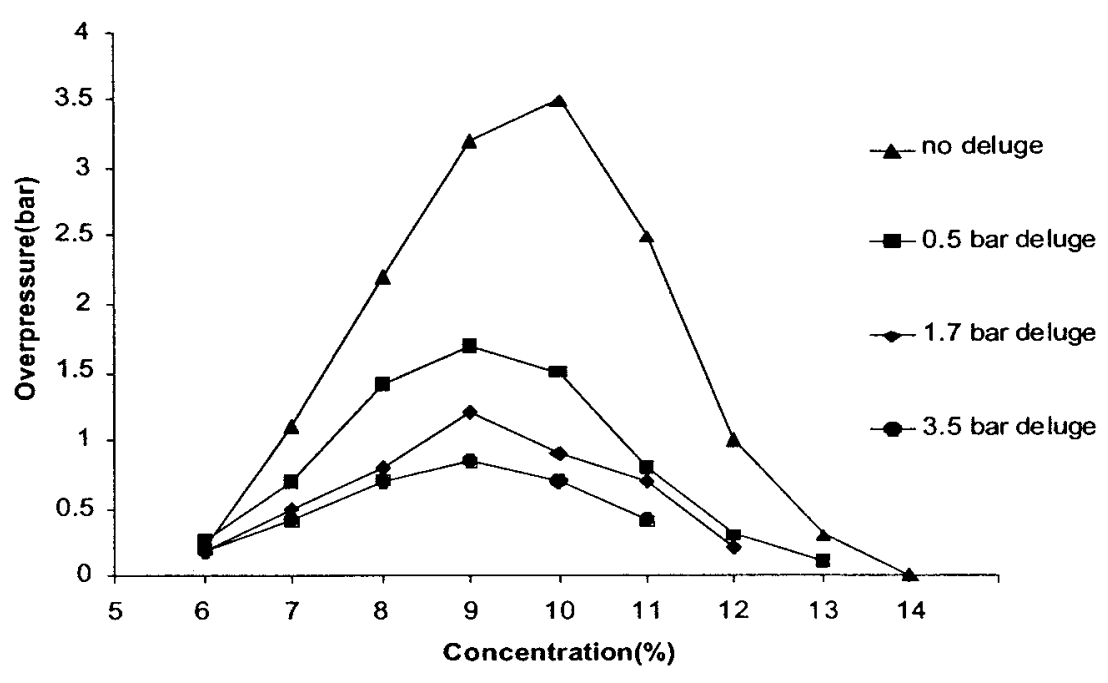

Fig. 3 Variation in the overpressure as a function of concentration

(a) when the water system is initiated and then fails prior to the gas concentration decreasing below the LFL and there is an ignition prior to the deluge failure;

(b) when the water spray functions throughout the critical time period and an ignition occurs.

Unmitigated explosions occur as follows:

(a) when the water spray system fails to activate when the gas is detected and ignition occurs;

(b) when the water spray activates but the gas ignites during the delay time (prior to mitigation);

(c) when the water spray system activates and is fully operational but then fails followed by ignition of the gas (mitigation not maintained).

The frequency of an explosion will take one of the following forms depending on whether the water spray initially activates:

$$
f_{\mathrm{e}}=P(\mathrm{~S}) \lambda_{\mathrm{L}} P(\mathrm{E}) P(\mathrm{WSFA})
$$

or

$$
f_{\mathrm{e}}=P(\mathrm{~S}) \lambda_{\mathrm{L}} P(\mathrm{E})[1-P(\mathrm{WSFA})]
$$

where $P(\mathrm{~S})$ is the probability of the event sequence represented by each event tree outcome, $\lambda_{\mathrm{L}}$ is the frequency of the gas leak, $P(\mathrm{E})$ is the probability of ignition and $P(\mathrm{WSFA})$ is the probability that the water system fails to activate.

Two methods have been considered to calculate the ignition probability $P(\mathrm{E})$ in equations (1) and (2). The first method is computationally efficient but requires strong assumptions regarding the gas dispersion. The second method is proposed to overcome these limitations but requires platform-specific CFD correlations to be produced.

\subsection{Method 1}

The method assumes that, as gas is released, it instantaneously mixes with the air. The mixing is assumed to be perfect, producing a uniform concentration of the gasin-air mixture throughout the module. This concentration is dependent upon the gas release rate, the relative volume of the module into which the gas is released and the ventilation rate. As long as the concentration is within the flammable limits and an ignition source occurs, an explosion is possible. In order to calculate the frequency of occurrence of an explosion it is necessary to know the frequency of occurrence of an ignition source. The ignition rate is assumed to be a constant, namely $\mu_{\mathrm{i}}$. This leads to occurrence times with a probability density function of

$$
f_{\mathrm{i}}(t)=\mu_{\mathrm{i}} \exp \left(-\mu_{\mathrm{i}} t\right)
$$

The fluid modelling provides the gas volume flowrate with respect to time into the module; by considering the ventilation flowrate, profiles of the type illustrated in Fig. 2 can be produced. When calculating the overpressure distribution of an explosion, the explosive range between the flammable limits is split into bands. This allows the frequency of an explosion at a certain concentration of gas to be determined. From the concentration at which ignition occurred, the British Gas empirical relationships yield the resulting overpressures generated for mitigated and unmitigated situations as shown in Fig. 3.

\subsection{Method 2}

The second method models the gas cloud build-up. The larger the gas cloud, the greater is the chance of encountering an ignition source. Instead of assuming perfect mixing of the gas and air in the module, CFD may 
be used to determine the concentration build-up of gas throughout the module with time. This will enable correlations for the time variation in the volume occupied by a specific concentration range of gas to be established.

The concentration contours produced as the gas enters the module will change position and shape with time. The volume encased within each concentration band will change with respect to time, as shown in Fig. 4 for a specific concentration band. The exact form of this graph will change for each scenario. In this example, the volume builds up rapidly at first owing to a fast release rate. Once the leak has exhausted, the volume reduces, as determined by the ventilation rate.

It is possible that the variation may be represented by a skewed function such as a log-normal function of the form

$$
V(t)=\frac{1}{\sigma t} \exp \left\{-\frac{1}{2}\left[\frac{\ln (t)-\varphi}{\sigma}\right]^{2}\right\}
$$

where $\varphi$ and $\sigma$ are the function parameters. Such a function is capable of representing the increase in volume as the gas is released and the decrease as ventilation dominates and the gas is exhausted.

To calculate the frequencies of explosions an ignition density is used rather than an ignition rate. In this case the probability of ignition is dependent on the ignition density and the dispersion and concentration of the gas cloud. For a specific concentration band $m$, the volume of gas follows a function $V_{m}(t)$. The ignition rate is then

$$
\lambda_{\mathrm{i}} V_{m}(t)
$$

where $\lambda_{\mathrm{i}}$ is the ignition rate per unit volume. This leads to a probability density function for the ignition source occurrence times of:

$$
f_{\mathrm{i}}(t)=\lambda_{\mathrm{i}} V_{m}(t) \exp \left[-\lambda_{\mathrm{i}} \int_{0}^{t} V_{m}(u) \mathrm{d} u\right]
$$

The modelling provides the times at which each concentration band develops and then disappears and the volume within this band. It is assumed that the lowest concentration band is the first to be reached and the last to be left, and that the highest is the last to be reached and the first to be left. Therefore, if $t_{\mathrm{E} m}$ is the time that band $m$ is entered and $t_{\mathrm{L} m}$ is the time that band $m$ is left, the following must apply:

$$
t_{\mathrm{E} 1}<t_{\mathrm{E} 2}<\cdots<t_{\mathrm{L} 2}<t_{\mathrm{L} 1}
$$

\section{CALCULATIONS}

The probability that ignition occurs within a specific concentration range is dependent upon the form of the ignition source probability function. Different calculation procedures result from the two methods described above.

\subsection{Ignition probability calculations for method 1}

When the form of the distribution of times between ignition source occurrences is given by equation (3), the following equations show how $P(\mathrm{E})$, the ignition probability, can be determined for a specific concentration band. For each concentration band there will be entering and leaving times; these will be denoted $t_{1 m}, t_{2 m}, t_{3 m}$ and $t_{4 m}$ if the upper limit of that concentration band is exceeded; otherwise, if the concentration begins to fall while in the band, there will be only $t_{1 m}$ and $t_{4 m}$, where $m$ refers to the specific concentration band. An ignition occurring in this band depends on which of the situations shown in Fig. 2 occurs. Ignition may only take place if it has not occurred previously within any of the concentration bands. For the case when the concentration band exceeds the UFL (Fig. 2c), the probability that ignition occurs in band $m$ is

$$
\begin{aligned}
P(\mathrm{E})= & P\left[\left(\text { ignition at time } v \text { between } t_{1 m} \text { and } t_{2 m}\right.\right. \\
& \text { AND no ignition between } \left.T_{1} \text { and } v\right) \text { OR } \\
& \text { (ignition at time } v \text { between } t_{3 m} \text { and } t_{4 m} \text { AND } \\
& \text { no ignition between } \left.\left.T_{1} \text { and } T_{2} \text { or } T_{3} \text { and } v\right)\right]
\end{aligned}
$$

Thus

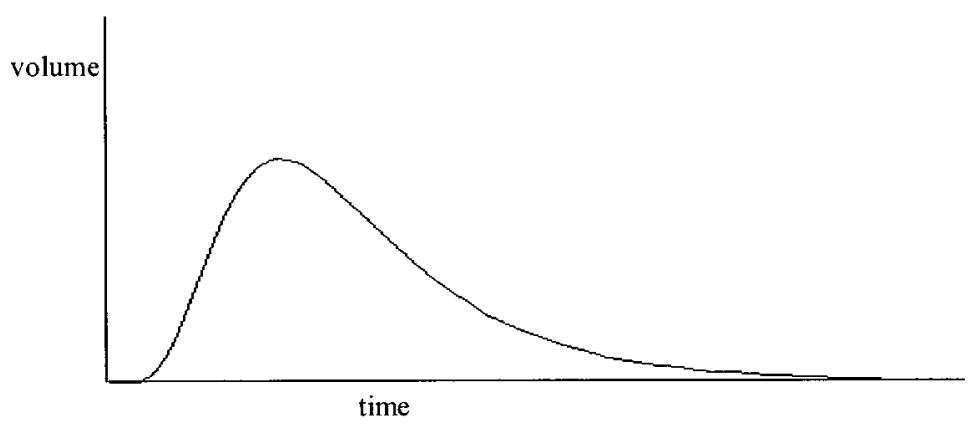

Fig. 4 Example of the expected growth and decay of the volume within a concentration band, using the log-normal function 


$$
\begin{aligned}
P(\mathrm{E})= & \int_{t_{1 m}}^{t_{2 m}} f_{\mathrm{i}}(v)\left[1-\int_{T_{1}}^{v} f_{\mathrm{i}}(u) \mathrm{d} u\right] \mathrm{d} v \\
& +\int_{t_{3 m}}^{t_{4 m}} f_{\mathrm{i}}(v)\left[1-\int_{T_{1}}^{T_{2}} f_{\mathrm{i}}(u) \mathrm{d} u-\int_{T_{3}}^{v} f_{\mathrm{i}}(u) \mathrm{d} u\right] \mathrm{d} v
\end{aligned}
$$

When the concentration does not exceed the UFL (Fig. 2b), $T_{2}$ and $T_{3}$ do not exist. There are two situations that could apply: firstly, the concentration may exceed the UFL of the specific band, therefore giving times $t_{1 m}$, $t_{2 m}, t_{3 m}$ and $t_{4 m}$; secondly, the concentration may exceed the LFL but not the UFL, only giving times $t_{1 m}$ and $t_{4 m}$. For the first situation, equation (9) reduces to

$$
\begin{aligned}
P(\mathrm{E})= & \int_{t_{1 m}}^{t_{2 m}} f_{\mathrm{i}}(v)\left[1-\int_{T_{1}}^{v} f_{\mathrm{i}}(u) \mathrm{d} u\right] \mathrm{d} v \\
& +\int_{t_{3 m}}^{t_{4 m}} f_{\mathrm{i}}(v)\left[1-\int_{T_{1}}^{v} f_{\mathrm{i}}(u) \mathrm{d} u\right] \mathrm{d} v
\end{aligned}
$$

For the second situation, equation (10) is modified to

$$
P(\mathrm{E})=\int_{t_{1 m}}^{t_{4 m}} f_{\mathrm{i}}(v)\left[1-\int_{T_{1}}^{v} f_{\mathrm{i}}(u) \mathrm{d} u\right] \mathrm{d} v
$$

Equations (9) to (11) give the ignition probability that can be substituted into equations (1) and (2) to yield the explosion frequency. If it is required to distinguish between the proportion of these explosions which are mitigated and those which are unmitigated, then equations (9) to (11) need to account for the failure, once running, of the deluge system.

Considering first the probability that the ignition occurs prior to activation of the water spray. This situation needs to account for the delay time from the detection of the gas to the initiation of the water spray and to the start of the deluge, namely $t_{\mathrm{d}}$. If $t_{\mathrm{d}}<t$, then this type of ignition cannot occur within that concentration band whereas, if $t_{\mathrm{d}}>t_{4_{m}}$, this type of ignition may take place at any time within the band; the probability of this is as in equation (9). The position of $t_{\mathrm{d}}$ relative to $t_{1 m}-t_{4 m}$ must be established, as for each case the probability equation will take the same form as equation (9), (10) or (11) with different limits of integration.

The probability that ignition occurs after the mitigation fails, given that it was activated, also depends on the delay time $t_{\mathrm{d}}$ and the failure frequency of the mitigation system. It is assumed that the failure rate of the water deluge system is constant, $\lambda_{\mathrm{ws}}$, leading to a failure density function

$$
f_{\mathrm{ws}}(u)=\lambda_{\mathrm{ws}} \exp \left(-\lambda_{\mathrm{ws}} u\right)
$$

To determine the probability of ignition in this case, the timing of the water spray delay must be considered to establish when such an unmitigated explosion could occur.

For $t_{\mathrm{d}}<t_{1 m}$, failure of the deluge system at time $u$ and occurrence of ignition at $v>u$, then the probability of an unmitigated ignition is

$$
\begin{aligned}
P(\mathrm{E})=\int_{t_{\mathrm{d}}}^{t_{1 m}} f_{\mathrm{ws}}(u)\left\{\int_{t_{1 m}}^{t_{2 m}} f_{\mathrm{i}}(v)\left[1-\int_{T_{1}}^{v} f_{\mathrm{i}}(s) \mathrm{d} s\right] \mathrm{d} v\right. \\
\left.+\int_{t_{3 m}}^{t_{4 m}} f_{\mathrm{i}}(v)\left[1-\int_{T_{1}}^{T_{2}} f_{\mathrm{i}}(s) \mathrm{d} s-\int_{T_{3}}^{v} f_{\mathrm{i}}(s) \mathrm{d} s\right] \mathrm{d} v\right\} \mathrm{d} u \\
+\int_{t_{1 m}}^{t_{2 m}} f_{\mathrm{ws}}(u)\left\{\int_{u}^{t_{2 m}} f_{\mathrm{i}}(v) \mathrm{d} v\left[1-\int_{T_{1}}^{v} f_{\mathrm{i}}(s) \mathrm{d} s\right]\right. \\
\left.+\int_{t_{3 m}}^{t_{4 m}} f_{\mathrm{i}}(v)\left[1-\int_{T_{1}}^{T_{2}} f_{\mathrm{i}}(s) \mathrm{d} s-\int_{T_{3}}^{v} f_{\mathrm{i}}(s) \mathrm{d} s\right] \mathrm{d} v\right\} \mathrm{d} u \\
+\int_{t_{2 m}}^{t_{3 m}} f_{\mathrm{ws}}(u)\left\{\int _ { t _ { 3 m } } ^ { t _ { 4 m } } f _ { \mathrm { i } } ( v ) \left[1-\int_{T_{1}}^{t_{2}} f_{\mathrm{i}}(s) \mathrm{d} s\right.\right. \\
\left.\left.\quad-\int_{T_{3}}^{v} f_{\mathrm{i}}(s) \mathrm{d} s\right] \mathrm{~d} v\right\} \mathrm{d} u \\
+\int_{t_{3 m}}^{t_{4 m}} f_{\mathrm{ws}}(u)\left\{\int _ { u } ^ { t _ { 4 m } } f _ { \mathrm { i } } ( v ) \left[1-\int_{T_{1}}^{T_{2}} f_{\mathrm{i}}(s) \mathrm{d} s\right.\right. \\
\left.\left.\quad-\int_{T_{3}}^{v} f_{\mathrm{i}}(s) \mathrm{d} s\right] \mathrm{~d} v\right\} \mathrm{d} u
\end{aligned}
$$

If $t_{\mathrm{d}}$ occurs at other points, then again the limits will change for the other situations.

An ignition when there is mitigation can occur in one of two ways. Either the water could function continuously throughout the flammable time duration or an ignition could occur at some point prior to its failure during this time period after its activation. As an example, if $t_{\mathrm{d}}<t_{1 m}$ and the ignition occurs at time $v$ prior to failure of the deluge system at time $u>v$, then the probability of a mitigated ignition is

$$
\begin{aligned}
P(\mathrm{E})=[1 & \left.-\int_{t_{\mathrm{d}}}^{t_{4 m}} f_{\mathrm{ws}}(u) \mathrm{d} u\right] \\
& \times\left\{\int_{t_{1 m}}^{t_{2 m}} f_{\mathrm{i}}(v)\left[1-\int_{T_{1}}^{v} f_{\mathrm{i}}(s) \mathrm{d} s\right] \mathrm{d} v\right. \\
& \left.+\int_{t_{3 m}}^{t_{4 m}} f_{\mathrm{i}}(v)\left[1-\int_{T_{1}}^{T_{2}} f_{\mathrm{i}}(s) \mathrm{d} s-\int_{T_{3}}^{v} f_{\mathrm{i}}(s) \mathrm{d} s\right] \mathrm{d} v\right\} \\
+ & \int_{t_{1 m}}^{t_{2 m}} f_{\mathrm{ws}}(u)\left\{\int_{t_{1 m}}^{u} f_{\mathrm{i}}(v)\left[1-\int_{T_{1}}^{v} f_{\mathrm{i}}(s) \mathrm{d} s\right] \mathrm{d} v\right\} \mathrm{d} u \\
+ & \int_{t_{2 m}}^{t_{3 m}} f_{\mathrm{ws}}(u)\left\{\int_{t_{1 m}}^{t_{2 m}} f_{\mathrm{i}}(v)\left[1-\int_{T_{1}}^{v} f_{\mathrm{i}}(s) \mathrm{d} s\right] \mathrm{d} v\right\} \mathrm{d} u \\
+ & \int_{t_{3 m}}^{t_{4 m}} f_{\mathrm{ws}}(u)\left\{\int_{t_{1 m}}^{t_{2 m}} f_{\mathrm{i}}(v)\left[1-\int_{T_{1}}^{v} f_{\mathrm{i}}(s) \mathrm{d} s\right] \mathrm{d} v\right. \\
& \left.+\int_{t_{3 m}}^{u} f_{\mathrm{i}}(v)\left[1-\int_{T_{1}}^{T_{2}} f_{\mathrm{i}}(s) \mathrm{d} s-\int_{T_{3}}^{v} f_{\mathrm{i}}(s) \mathrm{d} s\right] \mathrm{d} v\right\} \mathrm{d} u
\end{aligned}
$$

where the first term relates to the functioning of the water spray continuously over the time period and the following terms relate to failure of the water spray. For all other situations where $t_{\mathrm{d}}$ occurs at other time points $t_{\mathrm{d}} \geqslant t_{1 m}$, the limits will change as appropriate. The results gained by applying this approach have been 


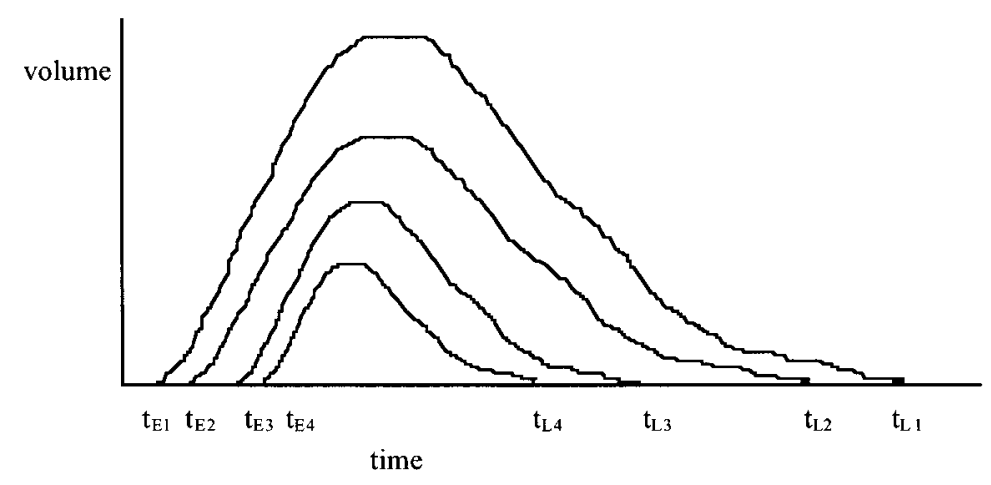

Fig. 5 Representation of the volume variation for each concentration band with time

derived and have been described in detail by Foster and Andrews [9].

\subsection{Ignition probability calculations for method 2}

As with method 1, this method assumes that an ignition will only occur at time $t$ if it has not occurred previously while a gas cloud existed which was within the explosive range. Method 1 involves integrating the probability density function for the ignition source using limits of integration that relate to the times of entry and exit for each concentration band over the flammable region. In this case, however, it is more complex. Each concentration range has its own probability density function that depends on the volume of the gas cloud within the concentration band limits. Therefore each time interval in equation (7), i.e. $t_{\mathrm{E} 1}$ to $t_{\mathrm{E} 2}, t_{\mathrm{E} 2}$ to $t_{\mathrm{E} 3}$, etc., must be considered. These are illustrated in Fig. 5 where the variation in the volume with time for each band is assumed not to intersect.

Consider first the probability of a gas cloud ignition at time $v$ :

$$
\begin{aligned}
P(\mathrm{E})= & \sum_{k=m}^{n-1} \int_{t_{\mathrm{E} k}}^{t_{\mathrm{E}(k+1)}} f_{\mathrm{i} m}(v)\left[1-\sum_{j=1}^{k} \int_{t_{\mathrm{E} j}}^{v} f_{\mathrm{ij}}(s) \mathrm{d} s\right] \mathrm{d} v \\
& +\int_{t_{\mathrm{E} n}}^{t_{\mathrm{L} n}} f_{\mathrm{i} m}(v)\left[1-\sum_{j=1}^{n} \int_{t_{\mathrm{E} j}}^{v} f_{\mathrm{ij}}(s) \mathrm{d} s\right] \mathrm{d} v \\
& +\sum_{k=m}^{n-1} \int_{t_{\mathrm{L}(k+1)}}^{t_{\mathrm{L} k}} f_{\mathrm{i} m}(v)\left[1-\sum_{j=k+1}^{n} \int_{t_{\mathrm{E} j}}^{t_{\mathrm{L} j}} f_{\mathrm{ij}}(s) \mathrm{d} s\right. \\
& \left.-\sum_{l=1}^{k} \int_{t_{\mathrm{E} l}}^{v} f_{\mathrm{i} l}(s) \mathrm{d} s\right] \mathrm{d} v
\end{aligned}
$$

where $n$ is the number of concentration bands, $P(\mathrm{E})$ is the probability of ignition while the concentration is in band $m$ and $f_{\mathrm{i} j}$ is the probability density function for the ignition source for band $j$. The first term considers the intervals between the entering times for each concentration band; e.g. if only the first concentration band has been reached, then it is unnecessary to consider the ignition density function relating to the other bands as ignition cannot yet occur within those ranges. However, as the gas release develops and the higher concentration bands are entered, more terms are added to account for the other ignition density functions of the other bands. The second term considers the time interval between entering and leaving the last band within the flammable limits. The final term accounts for the time intervals between the exit of each band which reduces the number of density functions that need to be considered.

To evaluate the proportions of these explosions that are unmitigated, the situation where the ignition happens before the water spray is activated needs to be considered. The calculations for this will be as in equation (15) with the limits changed to account for the time in the release at which the deluge activates.

Explosions occurring after the failure of the activated water spray depend on the activation time and the failure rate of the water spray system. The deluge failure rate is again assumed to be a constant, leading to the failure density function of equation (12). The activation time with respect to the limiting times of each band is considered.

For the case when $t_{\mathrm{d}}<t_{\mathrm{E} m}$, the probability of ignition in band $m$ at time $v$ is

$$
\begin{aligned}
P(\mathrm{E})= & \int_{t_{\mathrm{d}}}^{t_{\mathrm{E} m}} f_{\mathrm{ws}}(u)\left(\sum_{k=m}^{n-1}\left\{\int_{t_{\mathrm{E} k}}^{t_{\mathrm{E}(k+1)}} f_{\mathrm{i} m}(v)\left[1-\sum_{j=1}^{k} \int_{t_{\mathrm{E} j}}^{v} f_{\mathrm{i} j}(s) \mathrm{d} s\right] \mathrm{d} v\right\}+\int_{t_{\mathrm{E} n}}^{t_{\mathrm{L} n}} f_{\mathrm{i} m}(v)\left[1-\sum_{j=1}^{n} \int_{t_{\mathrm{E} j}}^{v} f_{\mathrm{ij}}(s) \mathrm{d} s\right] \mathrm{d} v\right. \\
& \left.+\sum_{k=m}^{n-1}\left\{\int_{t_{\mathrm{L}(k+1)}}^{t_{\mathrm{L} k}} f_{\mathrm{i} m}(v)\left[1-\sum_{j=k+1}^{n} \int_{t_{\mathrm{E} j}}^{t_{\mathrm{L} j}} f_{\mathrm{i} j}(s) \mathrm{d} s-\sum_{l=1}^{k} \int_{t_{\mathrm{E} l}}^{v} f_{\mathrm{i} l}(s) \mathrm{d} s\right] \mathrm{d} v\right\}\right) \mathrm{d} u \\
& +\sum_{j=m}^{n-1} \int_{t_{\mathrm{E} j}}^{t_{\mathrm{E}(j+1)}} f_{\mathrm{ws}}(u)\left(\sum_{k=j+1}^{n-1}\left\{\int_{t_{E k}}^{t_{E}(k+1)} f_{\mathrm{i} m}(v)\left[1-\sum_{l=1}^{k} \int_{t_{\mathrm{E} l}}^{v} f_{\mathrm{i} l}(s) \mathrm{d} s\right] \mathrm{d} v\right\}+\int_{t_{\mathrm{E} n}}^{t_{\mathrm{L} n}} f_{\mathrm{i} m}(v)\left[1-\sum_{l=1}^{n} \int_{t_{\mathrm{E} l}}^{v} f_{\mathrm{i} l}(s) \mathrm{d} s\right] \mathrm{d} v\right.
\end{aligned}
$$




$$
\begin{aligned}
& \left.+\sum_{k=m}^{n-1}\left\{\int_{t_{\mathrm{L}(k+1)}}^{t_{\mathrm{L} k}} f_{\mathrm{i} m}(v)\left[1-\sum_{l=k+1}^{n} \int_{t_{\mathrm{E} l}}^{t_{\mathrm{L} l}} f_{\mathrm{i} l}(s) \mathrm{d} s-\sum_{l=1}^{k} \int_{t_{\mathrm{E} l}}^{v} f_{\mathrm{il}}(s) \mathrm{d} s\right] \mathrm{d} v\right\}+\int_{u}^{t_{\mathrm{E}(j+1)}} f_{\mathrm{i} m}(v)\left[1-\sum_{l=1}^{j} \int_{t_{\mathrm{E} l}}^{v} f_{\mathrm{i} l}(s) \mathrm{d} s\right] \mathrm{d} v\right) \mathrm{d} u \\
& +\int_{t_{\mathrm{E} n}}^{t_{\mathrm{L} n}} f_{\mathrm{ws}}(u)\left(\int_{u}^{t_{\mathrm{L} n}} f_{\mathrm{i} m}(v)\left[1-\sum_{j=1}^{n} \int_{t_{\mathrm{E} j}}^{v} f_{\mathrm{i} j}(s) \mathrm{d} s\right] \mathrm{d} v\right. \\
& \left.+\sum_{k=m}^{n-1}\left\{\int_{t_{\mathrm{L}(k+1)}}^{t_{\mathrm{L} k}} f_{\mathrm{i} m}(v)\left[1-\sum_{j=k+1}^{n} \int_{t_{\mathrm{E} j}}^{t_{\mathrm{L} j}} f_{\mathrm{ij}}(s) \mathrm{d} s-\sum_{l=1}^{k} \int_{t_{\mathrm{E} l}}^{v} f_{\mathrm{il}}(s) \mathrm{d} s\right] \mathrm{d} v\right\}\right) \mathrm{d} u \\
& +\sum_{j=m}^{n-1} \int_{t_{\mathrm{L}(j+1)}}^{t_{\mathrm{L} j}} f_{\mathrm{ws}}(u)\left(\int_{u}^{t_{\mathrm{L} j}} f_{\mathrm{i} m}(v)\left[1-\sum_{l=1}^{j} \int_{t_{\mathrm{E} l}}^{v} f_{\mathrm{il}}(s) \mathrm{d} s-\sum_{l=j+1}^{n} \int_{t_{\mathrm{E} l}}^{t_{\mathrm{L} l}} f_{\mathrm{il}}(s) \mathrm{d} s\right] \mathrm{d} v\right. \\
& \left.+\sum_{k=m}^{j-1}\left\{\int_{t_{\mathrm{L}(k+1)}}^{t_{\mathrm{L} k}} f_{\mathrm{i} m}(v)\left[1-\sum_{l=k+1}^{n} \int_{t_{\mathrm{E} /}}^{t_{\mathrm{L} l}} f_{\mathrm{i} l}(s) \mathrm{d} s-\sum_{l=1}^{k} \int_{t_{\mathrm{E} l}}^{v} f_{\mathrm{il}}(s) \mathrm{d} s\right] \mathrm{d} v\right\}\right) \mathrm{d} u
\end{aligned}
$$

For all other intervals, equation (16) is used with a change of integration limits.

Similarly, for ignition occurring when the water spray is active, the probability of ignition when $t_{\mathrm{d}}<t_{\mathrm{E} m}$ is

$$
\begin{aligned}
& P(\mathrm{E})=\left[1-\int_{t_{\mathrm{d}}}^{t_{\mathrm{L} m}} f_{\mathrm{ws}}(u) \mathrm{d} u\right]\left(\sum_{k=m}^{n-1}\left\{\int_{t_{\mathrm{E} k}}^{t_{\mathrm{E}(k+1)}} f_{\mathrm{i} m}(v)\left[1-\sum_{j=1}^{k} \int_{t_{\mathrm{E} j}}^{v} f_{\mathrm{ij}}(s) \mathrm{d} s\right] \mathrm{d} v\right\}+\int_{t_{\mathrm{E} n}}^{t_{\mathrm{L} n}} f_{\mathrm{i} m}(v)\left[1-\sum_{j=1}^{n} \int_{t_{\mathrm{E} j}}^{v} f_{\mathrm{ij}}(s) \mathrm{d} s\right] \mathrm{d} v\right. \\
& \left.+\sum_{k=m}^{n-1}\left\{\int_{t_{\mathrm{L}(k+1)}}^{t_{\mathrm{L} k}} f_{\mathrm{i} m}(v)\left[1-\sum_{j=k+1}^{n} \int_{t_{\mathrm{E} j}}^{t_{\mathrm{L} j}} f_{\mathrm{ij}}(s) \mathrm{d} s-\sum_{l=1}^{k} \int_{t_{\mathrm{E} l}}^{v} f_{\mathrm{il}}(s) \mathrm{d} s\right] \mathrm{d} v\right\}\right) \\
& +\sum_{j=m}^{n-1} \int_{t_{\mathrm{E} j}}^{t_{\mathrm{E}(j+1)}} f_{\mathrm{ws}}(u)\left\{\int_{t_{\mathrm{E} j}}^{u} f_{\mathrm{i} m}(v)\left[1-\sum_{k=1}^{j} \int_{t_{\mathrm{E} k}}^{v} f_{\mathrm{i} k}(s) \mathrm{d} s\right] \mathrm{d} v+\sum_{l=m}^{j-1} \int_{t_{\mathrm{E} l}}^{t_{\mathrm{E}(l+1)}} f_{\mathrm{i} m}(v)\left[1-\sum_{p=1}^{l} \int_{t_{\mathrm{E} p}}^{v} f_{\mathrm{i} p}(s) \mathrm{d} s\right] \mathrm{d} v\right\} \mathrm{d} u \\
& +\int_{t_{\mathrm{E} n}}^{t_{\mathrm{L} n}} f_{\mathrm{ws}}(u)\left\{\int_{t_{\mathrm{E} n}}^{u} f_{\mathrm{i} m}(v)\left[1-\sum_{k=1}^{n} \int_{t_{\mathrm{E} k}}^{v} f_{\mathrm{i} k}(s) \mathrm{d} s\right] \mathrm{d} v+\sum_{l=m}^{n-1} \int_{t_{\mathrm{E} l}}^{t_{\mathrm{E}(l+1)}} f_{\mathrm{i} m}(v)\left[1-\sum_{p=1}^{l} \int_{t_{\mathrm{E} p}}^{v} f_{\mathrm{i} p}(s) \mathrm{d} s\right] \mathrm{d} v\right\} \mathrm{d} u \\
& +\sum_{j=m}^{n-1} \int_{t_{\mathrm{L}(j+1)}}^{t_{\mathrm{L} j}} f_{\mathrm{ws}}(u)\left(\int_{t_{\mathrm{L}(j+1)}}^{u} f_{\mathrm{i} m}(v)\left[1-\sum_{k=j+1}^{n} \int_{t_{\mathrm{E} k}}^{t_{\mathrm{L} k}} f_{\mathrm{i} k}(s) \mathrm{d} s-\sum_{l=1}^{j} \int_{t_{\mathrm{E} l}}^{v} f_{\mathrm{i} l}(s) \mathrm{d} s\right] \mathrm{d} v\right. \\
& +\sum_{k=m}^{n-1}\left\{\int_{t_{\mathrm{E} k}}^{t_{\mathrm{E}(k+1)}} f_{\mathrm{i} m}(v)\left[1-\sum_{l=1}^{k} \int_{t_{\mathrm{E} l}}^{v} f_{\mathrm{il}}(s) \mathrm{d} s\right] \mathrm{d} v\right\}+\int_{t_{\mathrm{E} n}}^{t_{\mathrm{L} n}} f_{\mathrm{i} m}(v)\left[1-\sum_{j=1}^{n} \int_{t_{\mathrm{E} j}}^{v} f_{\mathrm{ij}}(s) \mathrm{d} s\right] \mathrm{d} v \\
& \left.+\sum_{k=j+1}^{n-1}\left\{\int_{t_{\mathrm{L}(k+1)}}^{t_{\mathrm{L} k}} f_{\mathrm{i} m}(v)\left[1-\sum_{l=k+1}^{n} \int_{t_{\mathrm{E} l}}^{t_{\mathrm{L} l}} f_{\mathrm{i} l}(s) \mathrm{d} s-\sum_{l=1}^{k} \int_{t_{\mathrm{E} l}}^{v} f_{\mathrm{i} l}(s) \mathrm{d} s\right] \mathrm{d} v\right\}\right) \mathrm{d} u
\end{aligned}
$$

\section{DISCUSSION AND CONCLUSIONS}

Two methods to predict the explosion frequencies following a gas release on an offshore platform have been presented in this paper. In addition to the overall explosion frequency, both methods allow the gasin-air concentration and the deluge status to be identified at the point of ignition, thereby enabling the explosion frequencies to be linked to the resulting overpressures in the form of a distribution. This enables the determination of the frequency with which an explosion where overpressures exceed the design limit can be expected, and the design adequacy established. Typical results obtained from these methodologies have been given by Andrews et al. [2].

Other useful information which can be obtained from this type of analysis, particularly if conducted at the design stage, are the contributions to the overall or design limit explosion frequency of

(a) an explosion following a leak on a specified isolatable section,

(b) an explosion following the failure of any isolation valve or

(c) an explosion following the failure of any blowdown valve.

These quantities are known as importance measures and indicate the weak points in the system design and highlight where the most cost effective design changes can be made.

The two methods presented require different input data (e.g. ignition rate versus ignition rate density, or 
fixed cloud size versus time-variable cloud size). Dependent on the numerical values given to these factors, one model will not consistently provide higher predictions than the other (i.e. one method does not provide an upper bound).

The choice of the model applied will be dependent on the resources and information available to the analyst. The second method takes account of the variable gas cloud size due to the release rate decay and the effect of ventilation. To obtain such a correlation requires the platform module geometry to be considered together with a range of leak rates, ventilation rates and leak positions entailing a significant number of CFD runs. This approach, while requiring the additional work, is considered to have the potential to produce more accurate predictions.

The advantage of the first method presented is that it is less computationally intensive and requires more easily obtainable data. Its limiting assumption is that of perfect mixing. This has recently been shown to be valid, particularly for the higher release rates, by experimental work conducted by British Gas plc. In view of the uncertainties in much of the data available, this more efficient approach may provide an effective means by which decisions regarding the design adequacy of platforms may be influenced.

\section{REFERENCES}

1 Cullen, D. The Public Enquiry into the Piper Alpha Disaster, 1990 (HMSO, London).

2 Andrews, J. D., Smith, R. and Gregory, J. Procedure to calculate the explosion frequency for a module on an offshore platform. Trans. Instn Chem. Engrs, 1994, 72(B2), 69-82.

3 Billinton, R. and Allan, R. Reliability Evaluation of Engineering Systems, 2nd edition, 1992 (Plenum, New York).

4 Andrews, J. D. and Moss, T. R. Reliability and Risk Assessment, 1993 (Longman, London).

5 Cheers, F. Elements of Compressible Flow, 1963 (John Wiley, London).

6 Hanna, S. and Drivas, P. Guidelines for Use of Vapor Cloud Dispersion Models, 1987 (American Institute of Chemical Engineers, New York).

7 Catlin, C. A., Gregory, C. A. J., Johnson, D. M. and Walker, D. G. Explosion mitigation in offshore modules by general area deluge. Trans. Instn Chem. Engrs, 1993, 71(B2), 101-111.

8 Gregory, C. A. J. and Johnson, D. M. Explosions in offshore modules: assessment and research. In Proceedings of the Second International Conference on Offshore Structural Design, 1993.

9 Foster, K. J. and Andrews, J. D. Explosion modelling for offshore structures. In Proceedings of the European Safety and Reliability Conference (ESREL '98), Trondheim, Norway, June 1998. 